Check for updates

Cite this: Chem. Sci., 2019, 10, 4573 have been paid for by the Royal Society of Chemistry

Accepted 5th March 2019

DOI: $10.1039 / c 9 s c 00453 j$

rsc.li/chemical-science
๑ All publication charges for this article

Received 26th January 2019

\section{Aqueous acid-based synthesis of lead-free tin halide perovskites with near-unity photoluminescence quantum efficiency $\dagger$}

\author{
Aifei Wang, ${ }^{a}$ Yanyan Guo, ${ }^{a}$ Zhaobo Zhou, ${ }^{b}$ Xianghong Niu, ${ }^{c}$ Yonggang Wang, (D) d \\ Faheem Muhammad, ${ }^{a}$ Hongbo Li, (D) ${ }^{e}$ Tao Zhang, (D) Jinlan Wang, (D)*b \\ Shuming $\mathrm{Nie}$ (iD) *af and Zhengtao Deng (D) *a
}

\begin{abstract}
Recently, lead halide perovskites with outstanding emission performance have become new candidate materials for light-emitting devices and displays; however, the toxicity of lead and instability of halide perovskites remain significant challenges. Herein, we report the aqueous acid-based synthesis of highly emissive two-dimensional (2D) tin halide perovskites, (octylammonium) ${ }_{2} \mathrm{SnX}_{4}(\mathrm{X}=\mathrm{Br}$, I, or mixtures thereof), which displayed a high absolute photoluminescence (PL) quantum yield of near-unity in the solid-state, PL emission centered at $600 \mathrm{~nm}$ with a broad bandwidth $(136 \mathrm{~nm})$, a large Stokes shift (250 $\mathrm{nm})$, long-lived luminescence $(\tau=3.3 \mu \mathrm{s})$, and zero overlap between their absorption and emission spectra. Significantly, the stability study of $2 \mathrm{D}$ tin halide perovskites monitored by the PL quantum yield showed no changes after 240 days of storage at room temperature under ambient air and humidity conditions. The PL emission of the 2D tin halide perovskites was tuned from yellow to deep red by controlling halide composition. Furthermore, new yellow phosphors with superior optical properties are used to fabricate UV pumped white light emitting diodes (WLEDs). We expect these results to facilitate the development of new environmentally friendly and high-performance phosphors for future lighting and display technologies.
\end{abstract}

\section{Introduction}

In recent years, solid-state lighting technology has become a cost-competitive and energy-efficient alternative to conventional electrical lighting. ${ }^{1}$ This results in great interest in the study of the chemical synthesis, luminescence properties, and engineering technology of phosphor materials. Over the past few decades, the most studied highly fluorescent solid-state semiconductor phosphor materials involved toxic heavy metals,

${ }^{a}$ College of Engineering and Applied Sciences, Nanjing National Laboratory of Microstructures, Nanjing University, Nanjing, Jiangsu, 210093, P. R. China. E-mail: dengz@nju.edu.cn

${ }^{b}$ School of Physics, Southeast University, Nanjing 211189, P. R. China. E-mail: jlwang@seu.edu.cn

${ }^{c}$ School of Science, Nanjing University of Posts and Telecommunications, Nanjing 210046, People's Republic of China

${ }^{d}$ Center for High Pressure Science and Technology Advanced Research (HPSTAR), Beijing 100949, P. R. China

${ }^{e}$ College of Materials Science and Engineering, Beijing Institute of Technology, Haidian District, Beijing 100081, P. R. China

${ }^{f}$ Departments of Bioengineering, Chemistry, Electrical and Computer Engineering, and Materials Science and Engineering, University of Illinois at Urbana-Champaign, Urbana, IL, USA. E-mail: nies@illinois.edu

$\dagger$ Electronic supplementary information (ESI) available: Experimental section and characterization details, an additional SEM image, additional PL decay curves, and additional pictures. See DOI: 10.1039/c9sc00453j such as cadmium selenide (CdSe) and lead selenide ( $\mathrm{PbSe}$ ), which presents a serious threat to environmental and human health. ${ }^{2}$ Alternatively, indium phosphide (InP) and copper indium sulfide (CIS) were studied as heavy-metal-free alternatives, but their luminescence properties are not significant, and indium is not abundant in the earth, so its production cost or price is very high..$^{2-4}$ Recently, the outstanding performance of $\mathrm{APbX}_{3}$-type lead halide perovskites $\left(\mathrm{A}=\mathrm{Cs}^{+}, \mathrm{CH}_{3} \mathrm{NH}_{3}{ }^{+} ; \mathrm{X}=\mathrm{Cl}^{-}\right.$, $\left.\mathrm{Br}^{-}, \mathrm{I}^{-}\right)$in photovoltaic solar cells reignited a phenomenal interest to use them also for high-performance solid-state lighting. ${ }^{5-9}$ The main advantages making them particularly attractive are the excellent optical and optoelectronic properties, the facile synthetic process at low temperatures, the low densities of trap states, and the flexibility to tune the optical bandgaps in the whole visible region. ${ }^{10-14}$ Despite the rapid development of high-performance and low-cost lead halide perovskite materials, the problem of the long-term toxicity of lead has not been solved which needs to be addressed before their large-scale and extensive applications..$^{12,15}$

As a result, efforts are being made to explore new alternatives for heavy metal-free and earth abundant highly fluorescent semiconductor materials. ${ }^{16-19}$ In this regard, tin is one of the major industrial metals with low toxicity, but the oxidation of divalent $\mathrm{Sn}^{2+}$ in the lead-free tin halide perovskite structure generates spontaneous vacancies and thus compromises the 
device performance. ${ }^{20}$ To this end, a number of strategies to address this problem are reported. ${ }^{21-24}$ The use of tetravalent tin, such as perovskite derivative $\mathrm{Cs}_{2} \mathrm{SnI}_{6}$, as an alternative has been studied. ${ }^{22,23}$ We have also recently developed a strategy for the synthesis of highly stable $\mathrm{CsSnBr}_{3}$ nanocages using a perfluorooctanoic acid (PFOA) post-treatment strategy. ${ }^{24}$

A family of two-dimensional (2D) perovskites is recently attracting a great deal of attention and is described by the formula ( $\left.\mathrm{R}-\mathrm{NH}_{3}\right)_{2} \mathrm{~A}_{n-1} \mathrm{M}_{n} \mathrm{X}_{3 n+1}$, where $\mathrm{R}$ is alkyl chains, $\mathrm{A}$ is a small monovalent cation, $\mathrm{M}$ is a divalent metal and $\mathrm{X}$ is a halide. ${ }^{25,26}$ Such $2 \mathrm{D}$ perovskites are an attractive alternative to three-dimensional (3D) $\mathrm{APbX}_{3}$-type perovskites in terms of their tremendous advantages of tuning optoelectronic properties through both chemical and quantum-mechanical degrees of freedom. Each organic layer in such perovskites acts as a potential barrier confining the excitons within the $\mathrm{MX}_{6}$ octahedron layer; thereby, 2D perovskites exhibit good photoluminescence and electroluminescence even in bulk states. ${ }^{27-29}$ In addition, layered $2 \mathrm{D}$ perovskites have also been reported to be highly resistant to air and moisture as compared to their bulk counterparts because the hydrophobic alkyl chains block the layer of $\mathrm{MX}_{6}$ octahedrons and protect them from oxidation. ${ }^{30}$ Very recently, a $2 \mathrm{D}$ tin halide perovskite of $\left(\mathrm{C}_{18} \mathrm{H}_{35^{-}}\right.$ $\left.\mathrm{NH}_{3}\right)_{2} \mathrm{SnBr}_{4}$ with a QY of $88 \%$ has been reported using an organic based hot injection method, ${ }^{31}$ but the aqueous synthetic route to prepare $2 \mathrm{D}$ tin halide perovskites is still challenging due to the anticipated instability of metal halides in aqueous solution. Using solubility equilibrium between metal halides and their saturated ionic components, a 3D lead halide perovskite was synthesized in aqueous solution with a highest QY of $40 \%,{ }^{32}$ but the $2 \mathrm{D}$ counterparts displayed a rather low QY $(<10 \%) .{ }^{33,34}$ Despite the above-mentioned advances, the aqueous synthesis of highly fluorescent lead-free 2D metal halide perovskites has been rarely reported.

In this study, we report, for the first time, the preparation of highly emissive two-dimensional (2D) phase (OCTAm) ${ }_{2} \mathrm{SnX}_{4}$ (OCTAm = octylammonium cation) tin halide perovskites in acidic aqueous solution with ultra-high absolute photoluminescence (PL) quantum yield and ultra-high stability. In comparison to previous reports, the differences and significance of the current work are as follows: (i) this is the first report of the synthesis of highly fluorescent 2D tin halide perovskites with the emission centered at $600 \mathrm{~nm}$ with a PLQY of near-unity, large Stokes shifts, ultra-long lifetime, and good PL stability under ambient conditions. (ii) The entire synthesis process for 2D divalent tin halide perovskites was performed in acidic aqueous solution in an ambient air environment with high reproducibility and chemical yield (>80\%). (iii) The PL could be tuned from yellow to dark red through controlling halide composition. (iv) The new yellow phosphor with superior optical properties was employed to fabricate UV pumped white light emitting diodes (WLEDs).

\section{Results and discussion}

$2 \mathrm{D}$ tin perovskites with variable composition were prepared as described in the experimental section. Unlike previous reports wherein the synthesis process of tin-based perovskites required special care in inert gas environments, in our study the whole synthesis was performed in aqueous acid solution and ambient atmosphere without humidity and oxygen control, as shown in Scheme 1. Typically, we adopted octylamine as the starting cation agent and it was protonated and changed into an octylammonium (OCTAm) cation by acidification after being introduced into the system. The $2 \mathrm{D}$ tin perovskites were formed by a self-assembly driven crystallization process and stacked as organic-inorganic layers like 2D lead organic-inorganic perovskites. ${ }^{35}$

The crystalline nature of $2 \mathrm{D}$ perovskite crystals was confirmed by powder X-ray diffraction (PXRD) analysis. Powder X-ray diffraction (PXRD) measurements of (OCTAm) ${ }_{2} \mathrm{SnBr}_{4}$ showed periodic diffraction at low angles (Fig. 1a), and typical peaks resulting from atomic plane reflection of $2 \mathrm{D}$ perovskites at 8.9 and $13.2^{\circ}$ [the (002) and (003) planes] indicated the horizontal growth of these compounds. ${ }^{36}$ The presence of only (001) reflections in the XRD patterns suggests a 2D structure of (OCTAm $)_{2} \mathrm{SnBr}_{4}$, similar to that recently reported for this family of $(\mathrm{OAm})_{2} \mathrm{SnBr}_{4} \cdot{ }^{30}$ According to Bragg's law, the periodicity which corresponds to average layer spacing was calculated to be $19.94 \AA$. The anticipated structure of the $2 \mathrm{D}(\mathrm{OCTAm})_{2} \mathrm{SnBr}_{4}$ is shown in Fig. 1b, as the $\mathrm{SnBr}_{6}$ octahedral layer being separated by octylammonium. Fig. 1c shows the high-resolution transmission electron microscopy (HRTEM) image with the corresponding fast Fourier transform (FFT) of the 2D (OCTAm) ${ }_{2} \mathrm{SnBr}_{4}$ perovskites, and the interplanar spacing was determined to be $20 \AA$, which matches well with that of the (100) plane. The 2D (OCTAm) $)_{2} \mathrm{SnBr}_{4}$ perovskite layer spacing $(20.2 \AA)$ consists of a single metal halide octahedral layer which is $\sim 6.7$ $\AA$, and two interleaving OCTAm cation layers occupy a space of $13.5 \AA$ A. A layered morphology was observed in the scanning electron microscopy (SEM) images, as shown in Fig. 1d and S1. $\dagger$ The energy-dispersive X-ray spectroscopy (EDS, Fig. 1e and f) results suggest the homogeneous distribution of the elements of nitrogen, tin, and bromide in the (OCTAm) $)_{2} \mathrm{SnBr}_{4}$.

Since the procedure is so facile that the product can be readily scaled up with high reproducibility. The chemical yield of $2 \mathrm{D}$ (OCTAm) ${ }_{2} \mathrm{SnBr}_{4}$ perovskites was estimated to be $>80 \%$ based on the total Sn content using an ultra-large scale (over 8.5 gram) sample, which was produced in a single batch. Furthermore, the product showed a highly bright yellow PL under UV light. The optical properties of the $\mathrm{Sn}$ bromide perovskites were characterized via UV-vis absorption and PL spectroscopies

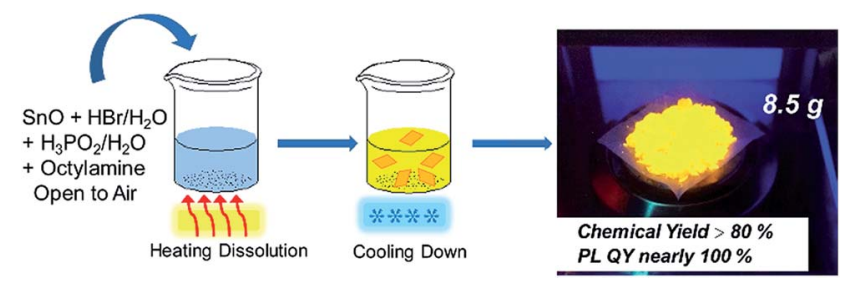

Scheme 1 Schematic representation of the synthesis of the 2D $(\mathrm{OCTAm})_{2} \mathrm{SnBr}_{4}$ by a facile aqueous acid-based synthetic method in ambient air. 

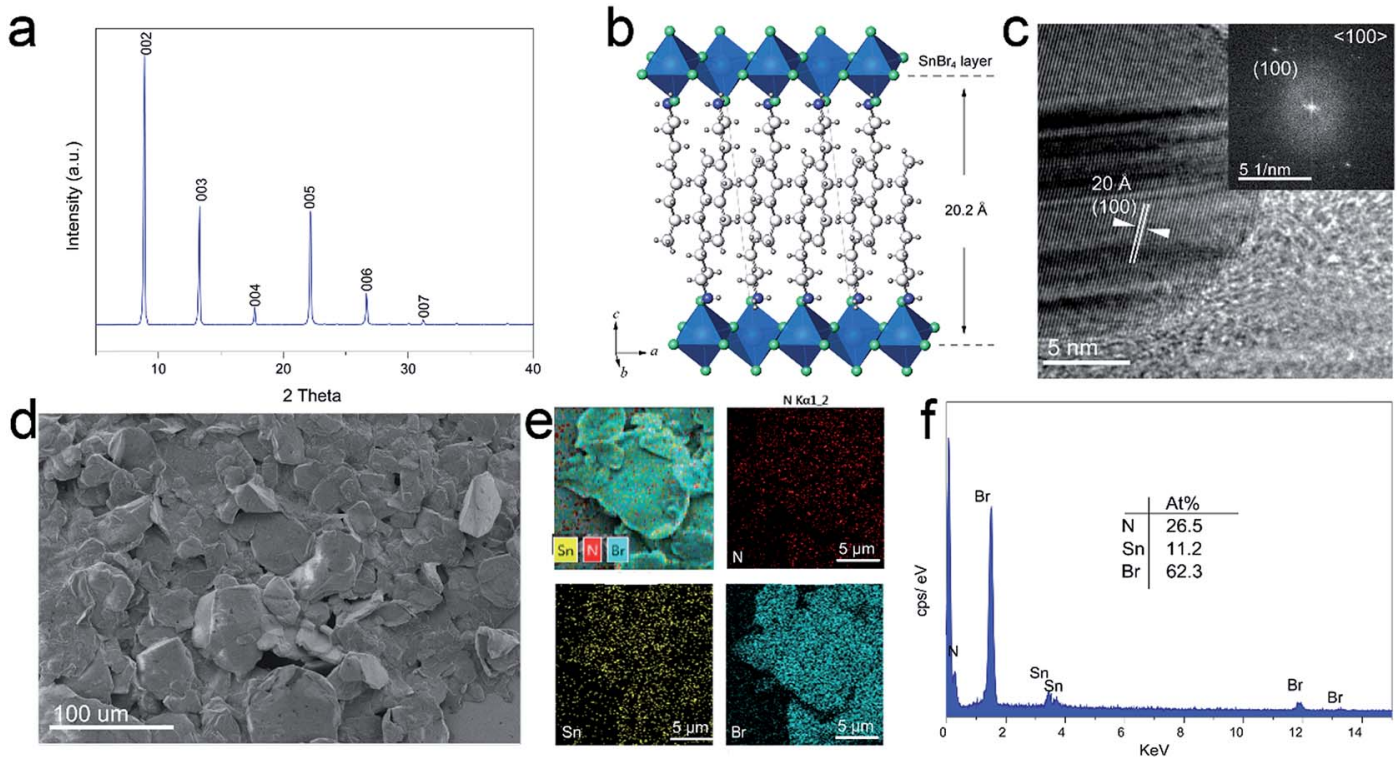

Fig. 1 The structure, morphology, and composition analysis of the as-synthesized $2 \mathrm{D}(\mathrm{OCTAm})_{2} \mathrm{SnBr}_{4}$. (a) Experimental powder $\mathrm{X}$-ray diffraction (PXRD) pattern. (b) Schematic representation of the crystal structure. (c) High-resolution TEM image, (d) scanning electron microscopy (SEM) image, (e) energy-dispersive X-ray spectroscopy (EDS) mapping image, and (f) the EDS spectrum of the $2 \mathrm{D}(\mathrm{OCTAm})_{2} \mathrm{SnBr}_{4}$ perovskites. Inset (c), the corresponding fast Fourier transform (FFT) image shown in (a).

(Fig. $2 \mathrm{~b}$ and c). The absorption and PL excitation results of 2D (OCTAm $)_{2} \mathrm{SnBr}_{4}$ display similar broad spectra from 300 to 400 $\mathrm{nm}$ centered at around $350 \mathrm{~nm}$. A plot of $[F(R) h \nu]^{2}$ versus energy indicates a direct band gap of $3.08 \mathrm{eV}$ (Fig. S2 $\dagger$ ). The PL emission spectrum shows a broad peak centered at $600 \mathrm{~nm}$ $(2.07 \mathrm{eV})$. Compared to the small Stokes shift and narrow emission caused by delocalized excitonic character in 3D perovskites, we observed the extra-large Stokes shift $(\sim 250 \mathrm{~nm}$,
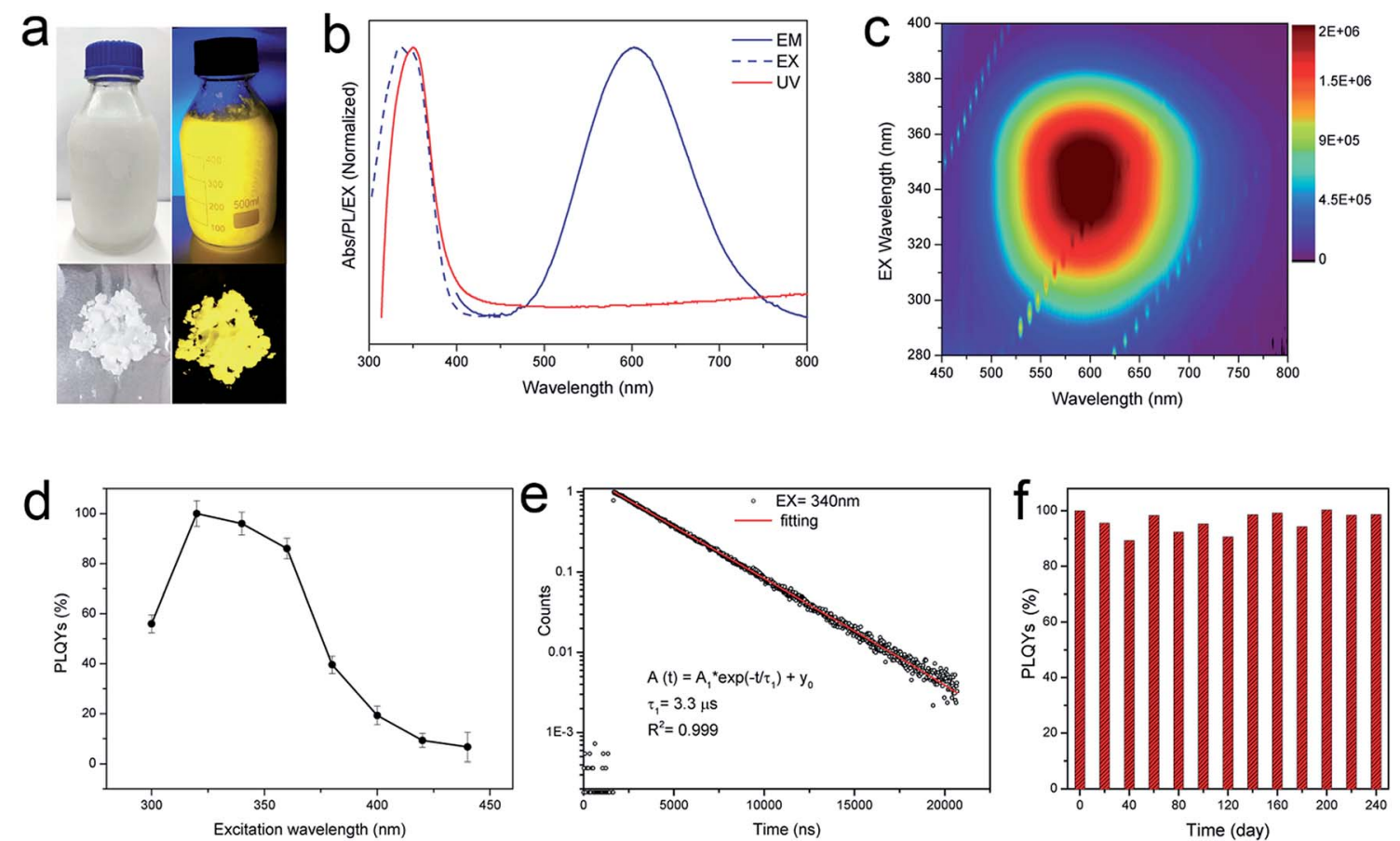

Fig. 2 Optical properties of 2D tin bromide perovskites. (a) Photograph of $0.5 \mathrm{~L}$ of 2D (OCTAm) ${ }_{2} \mathrm{SnBr}_{4}$ colloidal solution under room light (left) and UV light (right). (b) UV-vis absorption and PL excitation (EX) and PL emission spectra (EM) of the samples shown in (a). (c) Three-dimensional excitation-emission matrix (EEM) fluorescence spectrum. (d) The graph of absolute PL QYs under different excitation wavelengths. (e) Timeresolved $\mathrm{PL}$ decay and fitting curves of the $\mathrm{PL}$ emission at $600 \mathrm{~nm}$ with an excitation wavelength of $340 \mathrm{~nm}$. (f) Stability study of $2 \mathrm{D}$ $(\mathrm{OCTAm})_{2} \mathrm{SnBr}_{4}$ monitored by the PL QY changes as a function of exposure time (0-240 days in ambient air and humidity). 
i.e., $1.47 \mathrm{eV}$ ) and broad emission (full width at half-maximum, FWHM, $136 \mathrm{~nm}$, i.e., $467 \mathrm{meV}$ ) in 2D (OCTAm) ${ }_{2} \mathrm{SnBr}_{4}$ perovskites. According to previous reports, such a large Stokes shift and broad emission exist in both lead and tin 2D hybrid perovskites that stems from strong exciton couples in the metal-halide octahedral layer and creates transient elastic lattice distortions, viewed as self-trapping excitons. ${ }^{31,37} \mathrm{Simi}$ larly, we presume that the exciton self-trapping results in broad yellow emission in our (OCTAm) ${ }_{2} \mathrm{SnBr}_{4}$ materials. The $2 \mathrm{D}$ (OCTAm) $)_{2} \mathrm{SnBr}_{4}$ is further analyzed using three-dimensional excitation-emission matrix (EEM) fluorescence spectroscopy (Fig. 2c). There was no peak shift with the change of the excitation, suggesting that no other impurities or extra energy level existed in our samples.

$2 \mathrm{D}$ phase (OCTAm) $)_{2} \mathrm{SnBr}_{4}$ shows excitation dependent PL QYs and the absolute PL QY was near unity (95 $\pm 5 \%$ ) (Fig. 2d) in the solid-state. The PL QY of our 2D (OCTAm) ${ }_{2} \mathrm{SnBr}_{4}$ perovskite far exceeds the values reported in the case of aqueous synthesis of lead perovskites and is comparable with those of tin counterparts synthesized in organic solvents (Table S1†). It was found that there was a long PL lifetime decay of $3.3 \mu \mathrm{s}$ (Fig. 2e), which is similar to that of the $2 \mathrm{D}\left(\mathrm{C}_{18} \mathrm{H}_{35} \mathrm{NH}_{3}\right)_{2} \mathrm{SnBr}_{4}$ perovskites. The time-resolved PL decay curve recoded at a PL emission wavelength of $600 \mathrm{~nm}$ and an excitation wavelength of $340 \mathrm{~nm}$ was easily fitted to a single exponential decay function, which suggests that the PL decay is not related to the nonradiative processes, consistent with the high $\mathrm{PL} Q Y$ of the $2 \mathrm{D}$
$\left(\mathrm{C}_{18} \mathrm{H}_{35} \mathrm{NH}_{3}\right)_{2} \mathrm{SnBr}_{4} \cdot{ }^{38}$ Moreover, there was no noticeable change in the time-resolved PL decay curve under different excitation wavelengths of $280 \mathrm{~nm}$ and $365 \mathrm{~nm}$ (Fig. S3†). In order to investigate whether $\mathrm{H}_{3} \mathrm{PO}_{2}$ has any effect on the quantum efficiency of the product, we used different concentrations of acid during the synthesis of (OCTAm $)_{2} \mathrm{SnBr}_{4}$. Our findings ruled out any stabilization role of $\mathrm{H}_{3} \mathrm{PO}_{2}$ and no significant difference in quantum yield was observed (Fig. S4 $\dagger$ ), thus implying that the main function of $\mathrm{H}_{3} \mathrm{PO}_{2}$ is to avoid hydrolysis and oxidation of divalent tin. Stability of metal halide perovskites is critical for their further optical and optoelectronic applications. We found that the 2D (OCTAm) ${ }_{2} \mathrm{SnBr}_{4}$ exhibits high air-stability for over 240 days (Fig. 2f). It is not surprising that the (OCTAm) ${ }_{2} \mathrm{SnBr}_{4}$ perovskite was found to be very stable in ambient environment if we consider that the photoactive $\mathrm{Sn}-\mathrm{Br}$ octahedrons are well protected by octylammonium cations. Furthermore, we embedded (OCTAm) ${ }_{2} \mathrm{SnBr}_{4}$ into polystyrene (PS) films (Fig. S5$\mathrm{S} 7 \dagger$ ) that yielded a composite with superior optical clarity, bright emission (PL QY maximum $=80 \%$ ) and long lifetime $(3.3$ $\mu \mathrm{s})$. The high PL QY of the film is also related to the extra-large Stokes shifts, which would reduce re-absorption energy loss. These features are of vital importance for their future applications in solid-state lighting and luminescent solar concentrators (LSC). ${ }^{39}$

In addition to examining the tunability of 2D OCTAm-Sn hybrid perovskites, varying the halide ratio of the perovskites via in situ and post-synthesis strategies was studied. In situ protocol
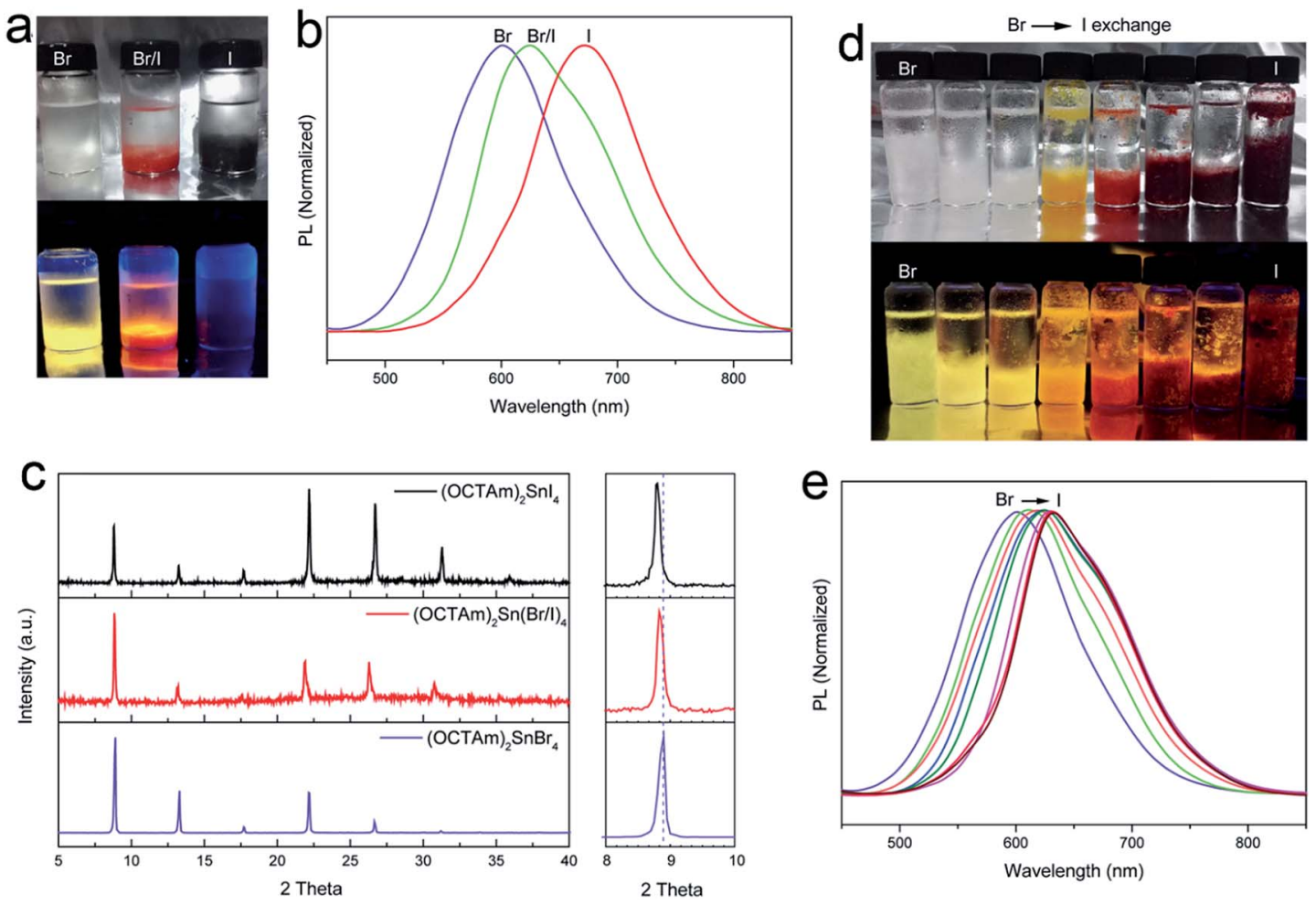

Fig. 3 Controllable photoluminescence via anion manipulation. $(\mathrm{a}-\mathrm{c})$ The direct synthesis of 2D phase tin halide perovskites by using different halide precursors. (a) Photographs of hybrid perovskites under room and UV light, from left to right $\mathrm{Sn}-\mathrm{Br}, \mathrm{Sn}-\mathrm{Br} / \mathrm{l}$ and $\mathrm{Sn}-\mathrm{I}$ perovskites. (b, c) PL spectra and PXRD patterns of the same perovskite samples. Right panel of (c) is the fine PXRD patterns from 8-10 ${ }^{\circ}$. (d, e) Photographs and PL spectra of the tin halide perovskites achieved by post-synthesis halide exchange of $(\mathrm{OCTAm})_{2} \mathrm{SnBr}_{4}$. 

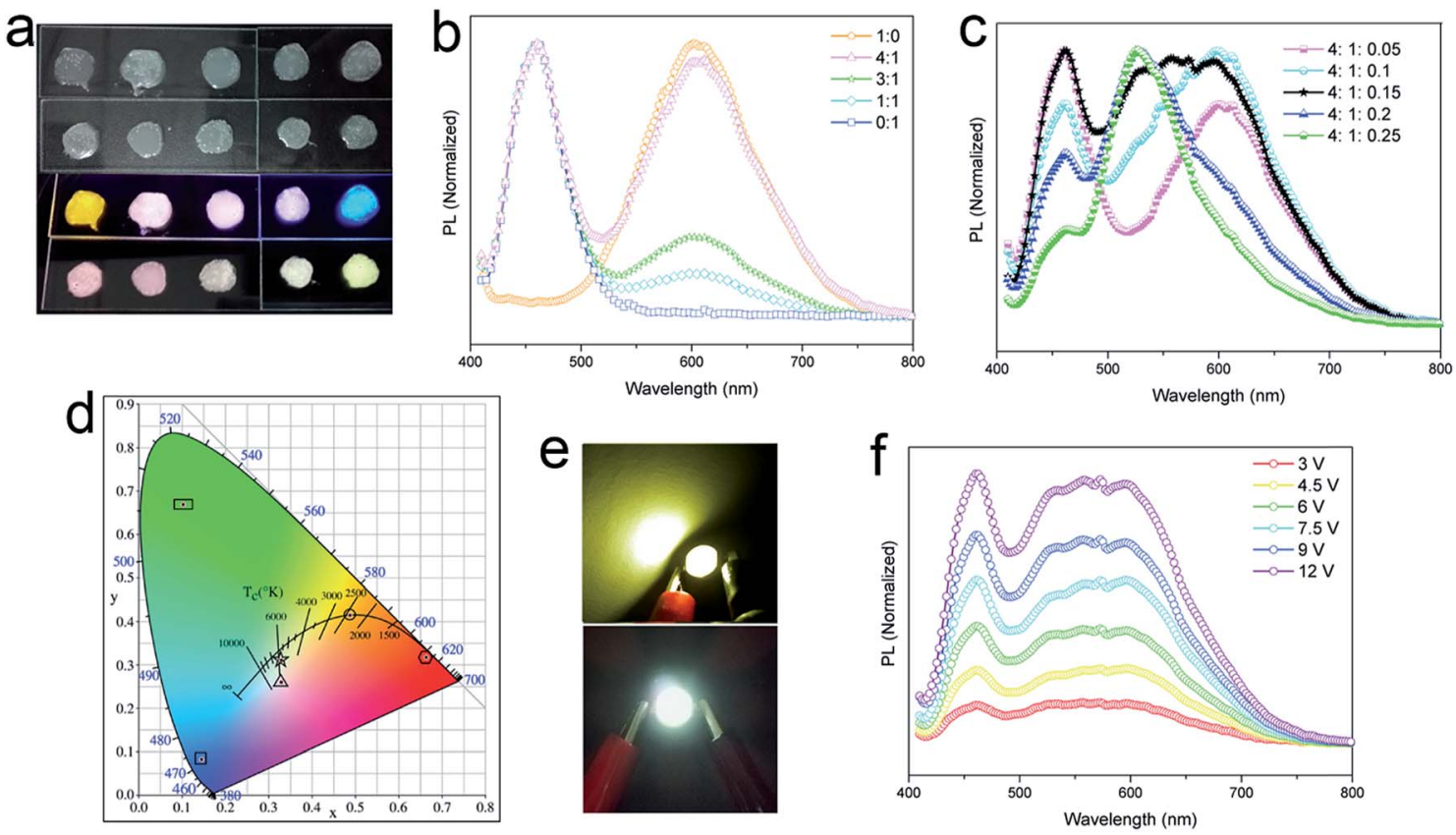

Fig. 4 Tin halide perovskites as the yellow phosphor for UV pumped white LEDs. (a) Images of yellow phosphors, blue/green phosphors, and their blends with different ratios embedded in PS films under sunlight (top panel) and $365 \mathrm{~nm}$ UV light (bottom panel). The first row in each panel are a yellow-blue phosphor mixture, and the second is a yellow-blue-green mixture. (b and c) PL emission spectra of different blending ratios: (b) blue and yellow phosphors and (c) yellow-blue-green phosphors. (d) Chromaticity coordinates of different ratios of the phosphor mixture plotted on the CIE1931 chromaticity chart: blue phosphor (square), yellow phosphor (round), yellow-blue phosphor $4: 1$ (triangle), and yellowblue-green phosphor $4: 1: 1.5$ (star). (e) UV (365 nm) pumped yellow and white LED devices. (f) PL spectra of a white LED at different driving voltages.

results in the generation of pure tin-iodide $(\mathrm{OCTAm})_{2} \mathrm{SnI}_{4}$ and the various compositions of tin-bromide/iodide (OCTAm) ${ }_{2} \mathrm{Sn}(\mathrm{Br} /$ I) ${ }_{4}$ are shown in Fig. $3 \mathrm{a}-\mathrm{c}$, which revealed that the PL emission spectrum can be tuned from $600 \mathrm{~nm}$ to $670 \mathrm{~nm}$ with a broad emission peak. The PL QY of (OCTAm $)_{2} \mathrm{SnI}_{4}$ was determined to be $41 \%$. Powder X-ray diffraction (PXRD) results also displayed periodic diffraction which verified a $2 \mathrm{D}$ layered perovskite structure in all products. The PXRD pattern of (002) planes from $\mathrm{Br}$ to I perovskites indicated a slight shift to lower angle due to tin-halide bond expansion.

The halide composition can also be regulated post-synthetically via an anion-exchange reaction using different halogen hydrides (Fig. 3b-e). In the anion-exchange process, pure bromide 2D OCTAm-Sn hybrid perovskites were used as the starting material; with increasing hydrogen iodide concentration the luminescence changes from yellow to deep red. This is consistent with previous reports about lead-based perovskite materials. ${ }^{40}$

The yellow-emitting phosphor of YAG:Ce ${ }^{3+}$ is widely used in commercial WLEDs, but the deficiency of red fluorescent components in YAG:Ce ${ }^{3+}$ (centered at $\sim 550 \mathrm{~nm}$, FWHM of 100 $\mathrm{nm}$ ) results in a low $(<80)$ color rendering index (CRI) in WLEDs. ${ }^{41}$ Rather than the addition of another red phosphor to broaden the emission band of the phosphor, use of any material with broad yellow emission covering also the red region can be a practical approach to improve the red component. Our lead-free 2D (OCTAm) $)_{2} \mathrm{SnBr}_{4}$ perovskites with high PL quantum yield and broadened yellow emission covering the red region motivated us to employ the obtained perovskite in WLEDs as a yellow-emitting phosphor. To validate the applicability of this material as a yellow phosphor, down conversion LEDs were fabricated. A commercial UV LED (365 nm) chip was used to optically pump different phosphor mixtures. The images of phosphor blended films and corresponding emission spectra are shown in Fig. 4a-c. A range of "warm" to "cold" white lights were attained by controlling the blending ratio between the three phosphors. The CIE color coordinates are shown in Fig. 4d. The UV pump LED of the single yellow phosphor showed a Correlated Colour Temperature (CCT) of $2500 \mathrm{~K}$, a CRI of 69 and CIE coordinates of $(0.488,0.415)$, while with a ratio of $4: 1$ by blue/yellow weight, a warm white emission with CIE coordinates of $(0.33,0.26)$, a CCT of 5635 and a CRI of 81 was achieved. A small amount of a green phosphor (yellow: blue : green $=4: 1: 1.5)$ helps tune to white emission with CIE coordinates of $(0.33,0.31)$, a CCT of $6530 \mathrm{~K}$, a CRI of 89 , and an LED lamp was fabricated based on this weight ratio (Fig. 4e). Various operating voltages were used to test the color stability of the white LED (Fig. 4f). With voltage increasing from $3 \mathrm{~V}$ to $12 \mathrm{~V}$, light intensity steadily increases without energy transfer between the three phosphors. Taken together, we envision the 2D (OCTAm $)_{2} \mathrm{SnBr}_{4}$ perovskite as a potential yellow phosphor for display backlight applications.

\section{Conclusions}

In summary, we have demonstrated facile aqueous acid-based synthesis of two-dimensional (OCTAm) ${ }_{2} \mathrm{SnBr}_{4}$ tin halide 
perovskites. The whole synthesis process was carried out in an ambient air and humidity environment, with high reproducibility and chemical yield. The obtained tin halide perovskites exhibited strong PL emission centered at $600 \mathrm{~nm}$ with broad FWHM (136 nm), large Stokes shifts (250 nm), high absolute PL quantum yield near unity in the solid-state, zero-overlap between their absorption and emission spectra, and an ultralong lifetime $(3.3 \mu \mathrm{s})$. These ultra-stable perovskite solid-state samples showed no changes after 240 days of storage at room temperature under ambient air and humidity conditions. These $2 \mathrm{D}$ tin perovskites act as yellow phosphors to produce optically pumped white LEDs. By a proper choice of raw sources and synthetic parameters, we anticipate that the present synthetic method can be extended for the rational design and synthesis of highly emissive, low-cost, environmentally benign, and stable metal halide perovskite phosphors, which will have broad prospects for application in the new generation of solid-state lighting and displays.

\section{Conflicts of interest}

There are no conflicts to declare.

\section{Acknowledgements}

The authors would like to thank Prof. Yuefeng Nie, Prof. Weiqiang Li, and Dr Yuguan Pan for help in acquiring the XRD spectra and helpful discussions regarding XRD analysis. This work was supported by the Natural Science Foundation of Jiangsu Province (Grant No. BK20180339 and BK20150581), the National Natural Science Foundation of China (Grant No. 51502130 and 21525311), the National Key R\&D Program of China (Grant No. 2017YFA0204800), the Thousand Talents Program for Young Researchers, the Shuangchuang Program of Jiangsu Province, Fundamental Research Funds for the Central Universities (Grant No. 021314380123), and the Jiangsu Key Laboratory for Nano Technology.

\section{Notes and references}

1 E. F. Schubert and J. K. Kim, Science, 2005, 308, 1274-1278.

2 J. Q. Grim, L. Manna and I. Moreels, Chem. Soc. Rev., 2015, 44, 5897-5914.

3 W. Shen, H. Tang, X. Yang, Z. Cao, T. Cheng, X. Wang, Z. Tan, J. You and Z. Deng, J. Mater. Chem. C, 2017, 5, 8243-8249.

4 H. Li, A. G. Kanaras and L. Manna, Acc. Chem. Res., 2013, 46, 1387-1396.

5 C. C. Stoumpos and M. G. Kanatzidis, Adv. Mater., 2016, 28, 5778-5793.

6 A. R. Srimath Kandada and A. Petrozza, Acc. Chem. Res., 2016, 49, 536-544.

7 L. Pedesseau, D. Sapori, B. Traore, R. Robles, H. H. Fang, M. A. Loi, H. Tsai, W. Nie, J. C. Blancon, A. Neukirch, S. Tretiak, A. D. Mohite, C. Katan, J. Even and M. Kepenekian, ACS Nano, 2016, 10, 9776-9786.
8 H. Tan, A. Jain, O. Voznyy, X. Lan, F. P. G. de Arquer, J. Z. Fan, R. Quintero-Bermudez, M. Yuan, B. Zhang and Y. Zhao, Science, 2017, 355, 722-726.

9 Y. Zhao and K. Zhu, Chem. Soc. Rev., 2016, 45, 655-689.

10 Y. Bekenstein, B. A. Koscher, S. W. Eaton, P. Yang and A. P. Alivisatos, J. Am. Chem. Soc., 2015, 137, 16008-16011.

11 Y. S. Park, S. Guo, N. S. Makarov and V. I. Klimov, ACS Nano, 2015, 9, 10386-10393.

12 W. Liu, Q. Lin, H. Li, K. Wu, I. Robel, J. M. Pietryga and V. I. Klimov, J. Am. Chem. Soc., 2016, 138, 14954-14961.

13 S. Sun, D. Yuan, Y. Xu, A. Wang and Z. Deng, ACS Nano, 2016, 10, 3648-3657.

14 C. Wang, Y. Zhang, A. Wang, Q. Wang, H. Tang, W. Shen, Z. Li and Z. Deng, Chem. Mater., 2017, 29, 2157-2166.

15 X. Yuan, S. Ji, M. C. De Siena, L. Fei, Z. Zhao, Y. Wang, H. Li, J. Zhao and D. R. Gamelin, Chem. Mater., 2017, 29, 80038011.

16 R. L. Z. Hoye, P. Schulz, L. T. Schelhas, A. M. Holder, K. H. Stone, J. D. Perkins, D. Vigil-Fowler, S. Siol, D. O. Scanlon, A. Zakutayev, A. Walsh, I. C. Smith, B. C. Melot, R. C. Kurchin, Y. Wang, J. Shi, F. C. Marques, J. J. Berry, W. Tumas, S. Lany, V. Stevanović, M. F. Toney and T. Buonassisi, Chem. Mater., 2017, 29, 1964-1988.

17 X. G. Zhao, J. H. Yang, Y. Fu, D. Yang, Q. Xu, L. Yu, S. H. Wei and L. Zhang, J. Am. Chem. Soc., 2017, 139, 2630-2638.

18 T. C. Jellicoe, J. M. Richter, H. F. Glass, M. Tabachnyk, R. Brady, S. E. Dutton, A. Rao, R. H. Friend, D. Credgington, N. C. Greenham and M. L. Bohm, J. Am. Chem. Soc., 2016, 138, 2941-2944.

19 P. V. Kamat, J. Bisquert and J. Buriak, ACS Energy Lett., 2017, 2, 904-905.

20 F. Wang, J. Ma, F. Xie, L. Li, J. Chen, J. Fan and N. Zhao, Adv. Funct. Mater., 2016, 26, 3417-3423.

21 K. P. Marshall, M. Walker, R. I. Walton and R. A. Hatton, Nat. Energy, 2016, 1, 16178.

22 A. Wang, X. Yan, M. Zhang, S. Sun, M. Yang, W. Shen, X. Pan, P. Wang and Z. Deng, Chem. Mater., 2016, 28, 8132-8140.

23 B. Lee, C. C. Stoumpos, N. Zhou, F. Hao, C. Malliakas, C.-Y. Yeh, T. J. Marks, M. G. Kanatzidis and R. P. H. Chang, J. Am. Chem. Soc., 2014, 136, 15379-15385.

24 A. Wang, Y. Guo, F. Muhammad and Z. Deng, Chem. Mater., 2017, 29, 6493-6501.

25 C. Huo, B. Cai, Z. Yuan, B. Ma and H. Zeng, Small Methods, 2017, 1, 1600018.

26 M. I. Saidaminov, O. F. Mohammed and O. M. Bakr, ACS Energy Lett., 2017, 2, 889-896.

27 P. Cheng, T. Wu, J. Zhang, Y. Li, J. Liu, L. Jiang, X. Mao, R. F. Lu, W. Q. Deng and K. Han, J. Phys. Chem. Lett., 2017, 8, 4402-4406.

28 L. Mao, W. Ke, L. Pedesseau, Y. Wu, C. Katan, J. Even, M. R. Wasielewski, C. C. Stoumpos and M. G. Kanatzidis, J. Am. Chem. Soc., 2018, 140, 3775-3783.

29 A. Biswas, R. Bakthavatsalam and J. Kundu, Chem. Mater., 2017, 29, 7816-7825.

30 L. Lanzetta, J. M. Marin-Beloqui, I. Sanchez-Molina, D. Ding and S. A. Haque, ACS Energy Lett., 2017, 2, 1662-1668. 
31 X. Zhang, C. Wang, Y. Zhang, X. Zhang, S. Wang, M. Lu, H. Cui, S. V. Kershaw, W. W. Yu and A. L. Rogach, ACS Energy Lett., 2018, 4, 242-248.

32 C. Geng, S. Xu, H. Zhong, A. L. Rogach and W. Bi, Angew. Chem., Int. Ed., 2018, 57, 9650-9654.

33 E. R. Dohner, A. Jaffe, L. R. Bradshaw and H. I. Karunadasa, J. Am. Chem. Soc., 2014, 136, 13154-13157.

34 T. Y. Li, W. A. Dunlap-Shohl, Q. W. Han and D. B. Mitzi, Chem. Mater., 2017, 29, 6200-6204.

35 L. Mao, Y. Wu, C. C. Stoumpos, M. R. Wasielewski and M. G. Kanatzidis, J. Am. Chem. Soc., 2017, 139, 5210-5215.

36 D. H. Cao, C. C. Stoumpos, O. K. Farha, J. T. Hupp and M. G. Kanatzidis, J. Am. Chem. Soc., 2015, 137, 7843-7850.

37 M. D. Smith and H. I. Karunadasa, Acc. Chem. Res., 2018, 51, 619-627.
38 C. Zhou, H. Lin, Y. Tian, Z. Yuan, R. Clark, B. Chen, L. J. van de Burgt, J. C. Wang, Y. Zhou, K. Hanson, Q. J. Meisner, J. Neu, T. Besara, T. Siegrist, E. Lambers, P. Djurovich and B. Ma, Chem. Sci., 2018, 9, 586-593.

39 C. S. Erickson, L. R. Bradshaw, S. McDowall, J. D. Gilbertson, D. R. Gamelin and D. L. Patrick, ACS Nano, 2014, 8, 34613467.

40 G. Nedelcu, L. Protesescu, S. Yakunin, M. I. Bodnarchuk, M. J. Grotevent and M. V. Kovalenko, Nano Lett., 2015, 15, 5635-5640.

41 W. Sun, Y. Jia, R. Pang, H. Li, T. Ma, D. Li, J. Fu, S. Zhang, L. Jiang and C. Li, ACS Appl. Mater. Interfaces, 2015, 7, 25219-25226. 